San Jose State University

From the SelectedWorks of Erin L. Woodhead

2016

\title{
Attitudes and Experiences With Older Adults: A Case for Service Learning for Undergraduates.
}

Hardeep K. Obhi, San José State University

Erin L. Woodhead 
Attitudes and Experiences with Older Adults:

A Case for Service Learning for Undergraduates 


\begin{abstract}
The current study examined whether relationship quality with older adults currently and in childhood, as well as experience with older adults, was associated with biases toward older adults and interest in working with older adults as a possible career area. We sampled undergraduate students $(N=753 ; M=18.97$ years, $S D=2.11$ years $)$ from a Northern California university. In hierarchical regression analyses, higher perceived quality of relationships with older adult family members, higher perceived social support, and lower perceived conflict from relationships with older adults was significantly associated with positive attitudes toward older adults. Interest in working with older adults was significantly associated with taking courses in aging, providing care to an older adult, and volunteering with older adults. These results suggest that positive relationships with older adults are useful in reducing biases, though student interactions with older adults are key in helping to promote interest in working with older adults. Keywords: attitudes toward aging, service learning, student attitudes, undergraduate education
\end{abstract}


Attitudes and Experiences with Older Adults:

A Case for Service Learning for Undergraduates

The projected increase in the aging population creates a demand for a workforce that is trained to work with older adults (Institute of Medicine, 2012). Despite this, it has proven difficult to attract students to the field of aging, potentially due to biases toward older adults or lack of exposure to aging-related content at the undergraduate level (Fitzgerald, Wray, Halter, Williams, \& Supiano, 2003; Hinrichsen, 2010). Prior research suggests that interest in working with older adults can be increased through positive personal experiences with older adults and exposure to aging-related content at different levels of training (Van Dussen \& Weaver, 2009). The current study examined how frequency and quality of current and childhood interactions with older adults was associated with biases toward older adults and intentions to work with this population. We also examined whether coursework and other informal experiences with older adults was associated with biases and interest toward this population.

\section{Student Biases Toward Older Adults}

Age-related stereotypes and general misunderstandings about older adults make it difficult for the workforce demand to be met (Slotterback \& Saarnio, 1996; Van Dussen \& Weaver, 2009). Although the majority of undergraduates have had some contact with older adults through interaction with older family members (Harwood, Hewstone, Paolini, \& Voci, 2005), many still use either negative or neutral terms to describe older adults (Sauer, 2006). For example, biases held by younger adults relate to the idea that older adults have lower levels of cognitive abilities than younger adults, live stationary lifestyles, and have problematic health conditions (Frenchs \& Mosher-Ashley, 2000; Slotterback \& Saarnio, 1996; Stuart-Hamilton \& 
Mahoney, 2003). Many undergraduate students also lack knowledge about the aging process in general (Kimuna, Knox, \& Zusman, 2005).

Some researchers have hypothesized that younger adults' negative views about older adults are due to younger adults focusing on ways in which they are different from older adults (Lovell, 2006). This leads to a more favorable view of younger than older adults, and perpetuates negative attitudes toward older adults. Some of these attitudes can be changed through positive interactions with older adults (Kalisch, Coughlin, Ballard, \& Lamson, 2013). Indeed, research tends to show that the more positive previous experiences individuals have with older adults, the more positive attitudes they hold about older adults. For example, previous experience with older adults increases general interest in older adults (Lee, Hoerr, Weatherspoon, \& Schiffman, 2007). Research also suggests that service learning has an impact on attitudes towards older adults. Service learning opportunities among undergraduates increase positive attitudes toward older adults, and help students gain a better understanding of older adults and aging (Gutheil, Chernesky, \& Sherratt, 2006).

\section{Student Interest in Working with Older Adults}

To start effectively building a workforce to provide services to older adults, researchers have to focus attention on variables that may increase or decrease interest in working with older adults. Among undergraduate students, frequent contact with older family members and taking an undergraduate course in psychology of aging were associated with interest in aging (Gorelik, Damron-Rodriguez, Funderburk, \& Solomon, 2000), although this study did not explicitly examine interest in working with older adults. In a study of undergraduate students, Robert and Mosher-Ashley (2000) found that students who cared for an older adult relative reported an increased desire to work with older adults as a career option. Moreover, Paton, Sar, Barber, and 
Holland (2001) found that more time spent in both formal and informal contact with the aging population was associated with an increased interest in wanting to work with older adults. Finally, Van Dussen and Weaver (2009) found that the more interaction and contact that undergraduates had with older adults, the more likely they were to have positive attitudes towards working with older adults as a prospective career area, though this study focused on the relevance of aging topics to various careers rather than asking directly about future career interests.

Studies of outcomes of service-learning projects suggest that these types of projects may increase interest in a career in aging. Undergraduates who engage in service learning with older adults tend to have an increased interest in further investigating the field of aging in terms of a possible career path (Vandsberger \& Wakefield, 2006). In light of the positive outcomes of service learning with older adults, it is possible that undergraduates who do not have frequent or positive interactions with older adults in their lives could obtain these experiences through service learning projects.

\section{Current Study}

The reviewed studies suggest that students' experiences with older adults and their exposure to educational material on the aging population may be important for increasing awareness about working with older adults. From our review of the literature, studies examining variables associated with increased interest in working with older adults have tended to focus on frequency of contact with older adults, to the exclusion of relationship quality variables, and have not consistently assessed intentions to work with older adults. To date, existing research on increasing interest in working with older adults among undergraduates has not examined relationship quality variables, has not examined multiple factors in the same model, or has not 
taken a comprehensive view of other factors that may be important in these associations. Instead, research has focused on frequency of contact (Gorelik et al., 2000; Luo, Zhou, Jin, Newman, \& Liang, 2013; Van Dussen \& Weaver, 2009) or caregiving for older adults (Robert \& MosherAshley, 2000). The current study examined multiple factors that may be important beyond contact and closeness, such as perceived relationship quality both currently and in childhood. We also examined whether these factors have different associations with attitudes toward older adults versus a desire to work with older adults in a future career.

In light of prior studies in this area, we hypothesized that more contact with older adults (measured via frequency of contact and other interactions) would be associated with more positive attitudes toward older adults and a greater intention to work with them in a future career. We also hypothesized that better relationship quality would be associated with more positive attitudes toward older adults and greater intentions to work with them. Although there is a lack of research on relationship quality and attitudes toward older adults, studies in the field of career decision-making suggest that attraction to a particular career may be influenced by positive personal interactions in that area (i.e., positive teaching and learning experiences; Watt et al., 2012).

\section{Method}

\section{Participants}

Participants were recruited from an Introductory Psychology course participant pool. They included 753 undergraduate students who ranged from the ages of 18 to $48(M=18.97$ years, $S D=2.11$ years). See Table 1 for further participant information.

\section{Measures}


Background questionnaire. This questionnaire was designed for the purposes of the study. Participants were asked about their age, gender, race/ethnicity, whether they were minoring in gerontology, how many classes they have had on aging issues, whether they have worked with older adults (paid or volunteer), whether they have ever provided care to an older adult, and general questions about whether they had a close relationship with an older adult while growing up or currently, and the overall quality of their relationships with older adult family and friends ( $1=$ low quality to $10=$ high quality). Participants were also asked about their interest in a career working with older adults ( $1=$ no interest to $10=$ very interested; $M=3.57, S D=2.14)$.

Aging Semantic Differential. The Aging Semantic Differential (ASD; Polizzi, 2003) is a questionnaire used to measure attitudes toward older adults. Participants are presented with 24 adjective pairs with seven ranking points between the adjectives (middle is neutral). Participants are asked to put a check mark along the continuum to indicate their attitude toward an adult over age 65. Scores over 96 indicate a general negative attitude toward older adults, while scores below this threshold indicate a generally positive attitude. The ASD has been shown to have high levels of reliability, with Cronbach's alphas ranging from .85 to .97 (Polizzi, 2003; Stewart, Eleazer, Boland, \& Wieland, 2007). The calculated alpha for the ASD in the current study was .94 and the mean was $72.75(S D=19.56)$.

Grandparent-Grandchild Relationship Questionnaire. A modified version of the Grandparent-Grandchild Relationship Questionnaire (GGRQ; Clingempeel, Colyar, Brand, \& Hetherington, 1992) was used to measure the degree of relationship involvement between older adults and participants. This measure is a composite of frequency of contact (physical involvement) and perceived closeness (emotional involvement), both currently and during childhood. The original version of the GGRQ includes three items, all of which ask participants 
to respond on a 5-point Likert scale about their current relationship with their grandparents. Items assess telephone/other media contact, face-to-face contact (both rated from $5=$ daily contact to $1=$ less than once a year $)$, and degree of emotional closeness $(5=$ extremely close to 1 $=$ not close at all). We added three additional questions to assess frequency of contact and emotional closeness in childhood. These three items regarding retrospective report of relationship involvement with older adults during childhood were added due to prior research by the second author suggesting that positive perceptions of older adults may develop partially due to childhood interactions with grandparents or other older adults (Woodhead et al., 2013). Items were examined at the individual level (possible range 1 to 5). Higher scores reflect a greater degree of relationship involvement. The reliability for the GGRQ tends to be reported per adult relative, with adequate Cronbach's alphas that range from .67 for grandfather-grandchild to .74 for grandmother-grandchild relationship (Clingempeel, Colyar, Brand, \& Hetherington, 1992). Across the different types of relatives assessed in the current study, the alpha for the GGRQ was .84. Table 2 presents the mean values for the GGRQ items for the current study.

Quality of Relationships Inventory. The Quality of Relationships Inventory (QRI; Pierce, Sarason, \& Sarason, 1991) was used to measure three dimensions of relationships with older adult relatives: social support, conflict, and depth. We selected specific items from the QRI that pertained to relationships with older adults. Specifically we selected four items from the social support subscale (example item: "To what extent can you turn to this person for advice about problems?"), six items from the conflict subscale (example item: "How critical of you is this person?"), and five items from the depth subscale (example item: "How significant is this relationship in your life?"). Participants are asked to respond on a 4-point Likert scale (1 = Not At All to $4=$ Very Much). Participants completed these items for each older adult relative they 
knew while growing up. Higher scores reflect more perceived social support, conflict, and depth among relationships with older adult relatives. Alpha coefficients for the QRI range from .88 for social support to .84 for the depth subscale (Pierce, Sarason, \& Sarason, 1991). The averaged QRI reliability for the subscales in the current study was .84. Table 2 presents the subscale scores for the QRI for the current study.

\section{Procedure}

The Institutional Review Board at San José State University approved the study. Participants completed the survey online and were awarded class credit for their participation. Once consent was obtained, participants completed the background questionnaire, which included two global questions about their quality of relationships with older adult family and friends, the ASD, the GGRQ, and the QRI. Unlike the background questionnaire which asked global questions about the quality of relationships with older adults, the GGRQ and QRI were completed separately for up to eight older adult relatives. Participants were first asked which relative they were reporting on (choices were: biological grandparent, step grandparent, great grandparent, aunt/great aunt, or uncle/great uncle) and were then asked to complete all the GGRQ and QRI items for that relative. They could then select up to seven other older adult relatives. They were asked to try and complete as many relative types as they had while growing up (up to eight; see Table 2 for mean number of relatives reported by participants).

\section{Analysis Plan}

First we examined completion rates for the full survey. Since not all participants had eight older adults relatives about whom to complete the GGRQ and QRI, we counted those items as complete if they answered all questions about at least one older adult relative, and indicated "did not have" for the remainder of the relative questions. Next examined were correlations of 
demographic variables (age, gender, ethnicity), experience with older adults (mean number of older adult relatives reported for the study, number of classes on aging, prior paid and volunteer work with older adults, prior caregiving for older adults), general questions about quality of relationships with older adults (current and past close relationship with an older adult, quality of relationship with older adult family members and friends), GGRQ items, and QRI subscales with the ASD (attitudes toward older adults) and interest in a career with older adults. Variables that were significantly correlated with the outcomes were retained and entered into hierarchical linear regression analyses, with the ASD total score and interest in a career with older adults as the outcomes. Regression analyses were used to address our hypotheses due to our interest in the strength of associations between contact and relationships with older adults and our outcomes of interest.

For the regression analyses, the background variables were entered in the first block and the relationship variables were entered in the second block. The tolerance and variance inflation factor (VIF) was examined for all variables in each regression and were acceptable (no variables with tolerance less than 0.10 or VIF greater than 10). Complete cases only were used for the regression analyses, which resulted in a loss of 140 cases. SPSS version 20 was used for all analyses, with an alpha of 0.05 .

\section{Results}

\section{Completion Rate}

Completion rate was calculated based on completion of all items included in analyses, including gender, ethnicity, age, prior exposure to work/classes with older adults, and all items of the GGRQ and QRI. The completion rate was $81.4 \%(n=613)$. Those who completed the survey were significantly more likely to be younger $(18.8$ years vs. $19.5 ; t(751)=3.41, p<0.001)$ and 
identify as Caucasian than other ethnicities $(90.6 \%$ completion rate vs. $79.2 \% ; \chi 2=12.08$, $p<0.001)$. There was no difference in completion rates by age, classes taken on aging, or having volunteered, provided caregiving for, or worked with older adults in the past.

\section{Correlations with Outcomes}

Pearson and Spearman correlations were used to examine associations of the demographic, experience, and relationship variables with the outcomes (Table 2). For the outcome of attitudes toward older adults (total ASD score), significantly more positive attitudes toward older adults were endorsed by those who worked with older adults in the past $(r=0.107)$, those who provided caregiving to an older adult $(r=0.085)$, those who had a close relationship with an older adult during childhood $(r=0.090)$, and those who reported higher quality relationships with older adult relatives $(r=-0.265)$ and friends $(r=-0.182)$. On the GGRQ, participants who had more positive attitudes toward older adults were significantly more likely to endorse a closer relationship with an older adult, both currently $(r=-0.182)$ and in childhood ( $r=-$ $0.184)$, and reported that they saw ( $r=-0.104)$ and talked to ( $r=-0.081)$ older adult relatives more frequently in childhood. All three of the QRI subscales were correlated with total ASD score. Participants who endorsed more social support ( $r=-0.232)$, less conflict $(r=0.157)$, and more depth $(r=-0.198)$ in relationships with older adults had significantly more positive attitudes toward them.

For the outcome of interest in a career working with older adults, significantly more interest was reported by those who had taken more classes on aging $(r=0.171)$, those who previously worked with $(r=-0.083)$, volunteered with $(r=-0.160)$, or provided care to older adults $(r=-0.166)$, and those who reported higher quality of relationship with older adult friends $(r=0.090)$. On the GGRQ, seeing older adult relatives $(r=0.106)$ and talking to them more in 
childhood ( $r=0.094)$ was significantly associated with more interest in working with them. On the QRI, those endorsing more depth in relationships with older adult relatives also reported significantly more interest in future work with older adults $(r=0.090)$.

\section{Regressions Examining Attitudes Toward and Interest in a Career with Older Adults}

More positive attitudes toward older adults were significantly associated with higher perceived quality of relationships with older adult family members $(\beta=-0.253)$, higher perceived social support $(\beta=-0.139)$ and lower perceived conflict $(\beta=0.157)$ from relationships with older adult family members, as measured by the QRI (Table 3). For this regression analysis, Block 1 explained $7.5 \%$ of the variance (adjusted $R^{2}=0.075$ ) and Block 2 explained $11.8 \%$ (adjusted $\left.R^{2}=0.118\right)$.

More interest in working with older adults was significantly associated with more classes on aging $(\beta=0.161)$, volunteering with older adults $(\beta=-0.110)$, and providing care to an older adult $(\beta=-0.129)$. None of the relationship variables were independently associated with interest in a career with older adults (Table 4). For this regression analysis, Block 1 explained $6.4 \%$ of the variance (adjusted $R^{2}=0.064$ ) and Block 2 explained $6.7 \%$ of the variance (adjusted $\left.R^{2}=0.067\right)$.

\section{Discussion}

The current study examined how multiple factors related to relationship quality with older adults may be important in reducing biases toward older adults and increasing undergraduates' interest in considering potential careers in working with older adults. Using regression analyses, we found that relationship quality variables were associated with significantly more positive attitudes toward older adults, whereas experience variables (classes, 
prior volunteer and caregiving work with older adults) were associated with significantly increased interest in a career working with older adults.

\section{Biases Toward Older Adults}

When controlling for other variables associated with biases toward older adults, we found that a global question about overall quality of relationships with older adult family members, as well as more social support and less conflict in relationships with older adults was significantly associated with more positive attitudes. These findings add new information to this literature due to our examination of quality of relationships as well as variables related to frequency of contact. Specifically, aspects of relationships with older adults, such as high social support and low conflict, appear to matter above and beyond frequency of contact. It is interesting to note that frequency of contact was not independently associated with improved attitudes toward older adults. It is possible that frequency of contact becomes insignificant when quality of relationships is taken into account. This should be examined in future studies, as our study is the only one we are aware of that considers both quality of relationships and frequency of contact with older adults.

One practical implication of this finding concerns the development of intergenerational service learning opportunities for undergraduates. Service learning opportunities have been successfully used to help undergraduate students understand and change their biases toward older adults (Hayslip, Caballero, Ward-Pinson, \& Riddle, 2013). Our results suggest that intergenerational service learning projects should be designed with particular attention to the quality of the interactions between the students and older adults. In addition to requiring a specific amount of time spent with the older adults, instructors might also consider routinely assessing the quality of interaction with older adults throughout the service learning project. In 
light of our findings of the importance of the quality of the relationship, it may be necessary to help students find a different opportunity if they are not having a positive experience in their initial volunteer assignment.

\section{Interest in Working with Older Adults}

When controlling for other variables associated with interest in working with older adults, we found that students who took more classes in aging, those who volunteered with older adults, and those who provided caregiving to older adults tended to have significantly higher levels of interest in a career working with older adults. Our research is in line with previous research studies, including Paton et al. (2001), Robert et al. (2000), and Van Dussen et al. (2009), in that that we also found that when participants took various classes in aging, they had significantly higher levels of interest in working with older adults. Our results extend these findings to suggest that other types of interactions with older adults may also increase a younger adult's interest in working with older adults.

Perhaps most interesting is that several key variables were not significantly associated with increased interest in working with older adults. Specifically, we did not find that frequency of contact was independently associated with interest in working with older adults, nor was the quality of the relationship. Although we were interested primarily in prior experiences with older adults predicting current attitudes and future career interest, it is likely that attitudes and behavioral experiences interact and are bi-directional. For instance, before considering a career with older adults, students may first need to either have or develop positive attitudes toward older adults. Our results suggest that positive attitudes could develop through high quality interactions with older people. After exposure to positive relationships with older adults, students may consider a career with older adults and seek out experiences that allow them to learn more 
about older adults though coursework or more meaningful interactions with older adults, such as through volunteering or caregiving.

\section{Limitations}

The primary limitation of our study is that it was designed as a correlational, retrospective study, and as such, our results do not speak to the issue of whether positive experiences with older adults precede the desire to work with older adults, or whether a desire to work with older adults leads students to seek out experiences with that population. Determining the temporal sequence of these events is important in better understanding how to draw students into gerontology if they do not have an initial interest in older adults. A further limitation is our use of a one-item measure to assess desire to work with older adults. Future studies may consider using a more comprehensive measure of intentions to work with this population. A final limitation is that our regression models were data driven and not conceptually based.

\section{Conclusions and Future Directions}

Our findings suggest a few possible educational interventions. If the ultimate goal is to attract students toward a potential career working with older adults, our results suggest that it could be worthwhile to require a course on adult development and aging, or at least ensure that lifespan development classes truly cover the full lifespan. Additionally, when designing intergenerational service learning opportunities, attention needs to be paid to variables beyond a specific time requirement for volunteering. Rather, establishment of a meaningful relationship with an older adult, regardless of the time commitment, appears to be particularly important to shaping interest in a career with older adults. In order to inform the design of intergenerational service learning projects, future research should employ a prospective research design to 
examine the influence of service learning experiences on career choices in working with older adults. 


\section{References}

Clingempeel, W. G., Colyar, J. J., Brand, E., \& Hetherington, E. M. (1992). Children's relationships with maternal grandparents: A longitudinal study of family structure and pubertal status effects. Child Development, 63, 1404-1422.

Fitzgerald, J. T., Wray, L. A., Halter, J. B., Williams, B. C., \& Supiano, M. A. (2003). Relating medical students' knowledge, attitudes, and experience to an interest in geriatric medicine. The Gerontologist, 43, 849-855. DOI: 10.1093/geront/43.6.849

Frenchs, E.M., \& Mosher-Ashley, P.M. (2000). College students' attitudes toward residential care facilities. Educational Gerontology, 26, 583-603. DOI: $10.1080 / 03601270050133919$

Gorelik, Y., Damron-Rodriguez, J., Funderburk, B., \& Solomon, D.H. (2000). Undergraduate interest in aging: Is it affected by contact with older adults? Educational Gerontology, 26, 623-638. DOI: 10.1080/03601270050200626

Gutheil, I. A., Chernesky, R. H., \& Sherratt, M. L. (2006). Influencing student attitudes toward older adults: Results of a service-learning collaboration. Educational Gerontology, 32, 771-784. DOI: 10.1080/03601270600835470

Harwood, J., Hewstone, M., Paolini, S., \& Voci, A. (2005). Grandparent-grandchild contact and attitudes toward older adults: Moderator and mediator effects. Personality and Social Psychology Bulletin, 31, 393-406. DOI: 10.1177/0146167204271577

Hayslip, B., Caballero, D., Ward-Pinson, M., \& Riddle, R. R. (2013). Sensitizing young adults to their biases about middle-aged and older persons: A pedagogical approach. Educational Gerontology, 39, 37-44. DOI: 10.1080/03601277.2012.660865 
Hinrichsen, G. A. (2010). Public policy and the provision of psychological services to older adults. Professional Psychology: Research and Practice, 41, 97. DOI: 10.1037/a0018643

Institute of Medicine (2012). The mental health and substance abuse work force for older adults: In whose hands? Retrieved from http://www.iom.edu/Reports/2012/The-Mental-Healthand-Substance-Use-Workforce-for-Older-Adults.aspx

Kalisch, H. R., Coughlin, D. R., Ballard, S. M., \& Lamson, A. (2013). Old age is a part of living: Student reflections on intergenerational service-learning. Gerontology \& geriatrics education, 34, 99-113. DOI: 10.1080/02701960.2012.753440

Kimuna, S. R., Knox, D., \& Zusman, M. (2005). College students' perceptions about older people and aging. Educational Gerontology, 31, 563-572. DOI:

$10.1080 / 03601270590962514$

Lee, S. Y., Hoerr, S. L., Weatherspoon, L., \& Schiffman, R. F. (2007). Previous experience with older adults positively affects nutrition students' attitudes toward this age group. Journal of Nutrition Education and Behavior, 39, 150-156. DOI: 10.1016/j.jneb.2006.08.029

Lovell, M. (2006). Caring for the elderly: changing perceptions and attitudes. Journal of Vascular Nursing, 24, 22-26. DOI:10.1016/j.jvn.2005.11.001

Luo, B., Zhou, K., Jin, E. J., Newman, A., \& Liang, J. (2013). Ageism among college students: A comparative study between US and China. Journal of Cross-Cultural Gerontology, 28, 49-63. DOI: 10.1007/s10823-013-9186-5

Paton, R. N., Sar, B. K., Barber, G., \& Holland, B. E. (2001). Working with older persons: Student views and experiences. Educational Gerontology, 27, 169-183. DOI: $10.1080 / 03601270151075589$ 
Pierce, G. R., Sarason, I. G., \& Sarason, B. R. (1991). General and relationship-based perceptions of social support: are two constructs better than one? Journal of Personality and Social Psychology, 61, 1028-1039. DOI: 10.1037/0022-3514.61.6.1028

Polizzi, K. G. (2003). Assessing attitudes toward the elderly: Polizzi's refined version of the aging semantic differential. Educational Gerontology, 29, 197-216. DOI: $10.1080 / 713844306$

Robert, R., \& Mosher-Ashley, P. M. (2000). Factors influencing college students to choose careers working with elderly persons. Educational Gerontology, 26, 725-736. DOI: $10.1080 / 036012700300001386$

Sauer, B. L. (2006). Student-directed learning in a community geriatrics advanced pharmacy practice experience. American Journal of Pharmaceutical Education, 70, 54.

Slotterback, C. S., \& Saarnio, D. A. (1996). Attitudes toward older adults reported by young adults: Variation based on attitudinal task and attribute categories. Psychology and Aging, $11,563$.

Stewart, T. J., Eleazer, G. P., Boland, R., \& Wieland, G. D. (2007). The middle of the road: Results from the aging semantic differential with four cohorts of medical students. Journal of the American Geriatrics Society, 55, 1275-1280. DOI: 10.1111/j.15325415.2007.01319.x

Stuart-Hamilton, I., \& Mahoney, B. (2003). The effect of aging awareness training on knowledge of, and attitudes towards, older adults. Educational Gerontology, 29, 251-260. DOI: $10.1080 / 713844305$ 
Van Dussen, D. J., \& Weaver, R. R. (2009). Undergraduate students' perceptions and behaviors related to the aged and to aging processes. Educational Gerontology, 35, 342-357. DOI: $10.1080 / 03601270802612255$

Vandsberger, E., \& Wakefield, M. (2006). Service learning with rural older adults: Effects on students' career perspectives in gerontology. Journal of Intergenerational Relationships, 3, 83-97. DOI: 10.1300/J194v03n04_06

Watt, H. M. G., Richardson, P. W., Klusmann, U., Kunter, M., Beyer, B., Trautwein, U., \& Baumert, J. (2012). Motivations for choosing teaching as a career: An international comparison using the FIT-Choice scale. Teaching and Teacher Education, 28, 791-805. DOI: $10.1016 /$ j.tate .2012 .03 .003

Woodhead, E. L., Emery, E. E., Pachana, N. A., Scott, T. L., Konnert, C. A., \& Edelstein, B. A. (2013). Graduate students' geropsychology training opportunities and perceived competence in working with older adults. Professional Psychology: Research and Practice, 44, 355-362. DOI: 10.1037/a0034632 
Table 1

Participant Characteristics

Variable Percentage

\section{Ethnicity}

Asian

40.5

Hispanic

28.6

White

24.2

African American

Pacific Islander

Other

0.8

Age

$18-20$

91.4

$21-23$

6.2

24-26

1.5

$27+$

Gender

Male

Female

55.8

Are you considering a gerontology minor? (Yes)

$2.1 \%$

Have you worked with older adults currently or in the past? (Yes)

$74.5 \%$

Provided care to an older adult friend or family member? (Yes)

$74.4 \%$

Volunteered with older adults? (Yes)

$46.1 \%$

Note. $N=753$ 
Table 2

Means and Correlations with Outcomes

\begin{tabular}{llll}
\hline Variable & Mean (SD) or & Attitudes & Interest in Career \\
& Percentage & Toward Older & with Older Adults \\
& & & \\
& & Adults (ASD) & \\
& &
\end{tabular}

Demographic Characteristics

Ethnicity

$-$

$-0.043$

0.005

Gender $^{\mathrm{c}}$

0.037

$-0.016$

Age

$18.85(1.37)$

0.009

0.019

Experience with Aging/Older Adults

Number of relatives reported for study ${ }^{\mathrm{d}}$

$4.72(2.28)$

$-0.026$

$-0.002$

Number of classes on aging

$1.61(0.87)$

$-0.045$

$0.171 * * *$

Prior paid work with older adults? ${ }^{\mathrm{e}}$

-- $0.107 * * \quad-0.083^{*}$

Prior volunteer work with older adults? ${ }^{\mathrm{e}}$

$--$

0.029

$-0.160 * * *$

Prior caregiving for older adults?

$--$

$0.085^{*}$

$-0.166^{* * *}$

Overall Relationship Quality

Current close relationship?

$88.6 \%$ Yes

0.065

$-0.060$

Close relationship in childhood?

$85.3 \%$ Yes

$0.090 *$

$-0.032$

Quality of relationships: Older adult family

$7.94(2.14)$

$-0.265^{* * *}$

0.052

Quality of relationships: Older adult friends

$6.99(2.52)$

$-0.182 * * *$

$0.090^{*}$

Frequency of Contact and Closeness

GGRQ current closeness

GGRQ closeness in childhood

GGRQ current frequency seeing

GGRQ childhood frequency seeing
$3.20(1.00)$

$-0.182 * * *$

0.047

$3.29(0.93)$

$-0.184 * * *$

0.047

$2.32(0.94) \quad-0.044$

0.066

2.94 (1.08)

$-0.104 * *$

$0.106^{* *}$ 
GGRQ current frequency talking

GGRQ childhood frequency talking

Quality of Relationships

QRI Social Support

QRI Conflict

QRI Depth
$2.34(0.94)$

$2.64(1.10)$

$-0.060$

0.045

$-0.081^{*}$

$0.094^{*}$

$2.55(0.74)$

$-0.232 * * *$

0.051

$1.60(0.48)$

$0.157 * * *$

0.075

$2.50(0.62)$

$-0.198 * * *$

$0.090^{*}$

Note. $N=613$.

${ }^{\mathrm{a}}$ For the ASD, lower scores indicate more positive attitudes toward older adults.

${ }^{\mathrm{b}}$ For interest in a career with older adults, higher scores indicate more interest.

${ }^{\mathrm{c}}$ Gender coded as $1=$ male, $2=$ female.

${ }^{\mathrm{d}}$ This variable represents the mean of the number of older adult relatives for whom the participant completed the GGRQ and QRI.

${ }^{\mathrm{e}}$ Yes/No questions coded as $1=$ yes, $2=$ no.

$* p<0.05, * * p<0.01, * * * p<0.001$. 
Table 3

Regressions Examining Attitudes Toward Older Adults ${ }^{a}$

\begin{tabular}{|c|c|c|c|c|}
\hline Variable & $B$ & $S E B$ & $\beta$ & $t$ \\
\hline \multicolumn{5}{|l|}{ Block 1} \\
\hline Paid work with older adults ${ }^{\mathrm{b}}$ & 2.920 & 1.806 & 0.065 & 1.617 \\
\hline Caregiving $^{\mathrm{b}}$ & 2.071 & 1.818 & 0.046 & 1.140 \\
\hline Close relationship in childhood ${ }^{b}$ & -3.119 & 2.462 & -0.056 & -1.267 \\
\hline Quality of relationships: older adult family & -2.316 & 0.447 & -0.253 & $-5.187 * * *$ \\
\hline Quality of relationships: older adult friends & -0.431 & 0.350 & -0.056 & -1.233 \\
\hline \multicolumn{5}{|l|}{ Block 2} \\
\hline GGRQ current closeness & 0.182 & 1.091 & 0.009 & 0.167 \\
\hline GGRQ closeness in childhood & -0.311 & 1.208 & -0.015 & -0.258 \\
\hline GGRQ childhood frequency seeing & -1.364 & 0.958 & -0.075 & -1.423 \\
\hline GGRQ childhood frequency talking & 0.903 & 0.925 & 0.051 & 0.977 \\
\hline QRI Social Support & -3.655 & 1.447 & -0.139 & $-2.526 * *$ \\
\hline QRI Conflict & 6.357 & 1.619 & 0.157 & $3.927 * * *$ \\
\hline QRI Depth & -1.899 & 1.946 & -0.060 & -0.976 \\
\hline
\end{tabular}

Notes. $N=613$.

Block 1 Adjusted $R^{2}=0.075$, Block 2 Adjusted $R^{2}=0.118$.

${ }^{\mathrm{a}}$ For the outcome of attitudes toward older adults, lower scores indicate more positive attitudes.

${ }^{\mathrm{b}}$ Yes/No questions coded as $1=$ yes, $2=$ no.

$* * p<0.01, * * * p<0.001$ 
Table 4

Regressions Examining Interest in Working With Older Adults ${ }^{a}$

\begin{tabular}{|c|c|c|c|c|}
\hline Variable & $B$ & $S E B$ & $\beta$ & $t$ \\
\hline \multicolumn{5}{|l|}{ Block 1} \\
\hline Number of classes on aging & 0.398 & 0.098 & 0.161 & $4.081 * * *$ \\
\hline Paid work with older adults ${ }^{b}$ & 0.077 & 0.209 & 0.016 & 0.370 \\
\hline Volunteer work with older adults ${ }^{b}$ & -0.475 & 0.183 & -0.110 & $-2.597 * *$ \\
\hline Caregiving $^{\mathrm{b}}$ & -0.637 & 0.202 & -0.129 & $-3.151 * *$ \\
\hline Quality of relationships: older adult friends & 0.062 & 0.034 & 0.073 & 1.835 \\
\hline \multicolumn{5}{|l|}{ Block 2} \\
\hline GGRQ childhood frequency seeing & 0.150 & 0.108 & 0.075 & 1.391 \\
\hline GGRQ childhood frequency talking & 0.042 & 0.104 & 0.021 & 0.400 \\
\hline QRI Depth & 0.234 & 0.183 & 0.068 & 1.280 \\
\hline
\end{tabular}

Notes. $N=613$.

Block 1 Adjusted $R^{2}=0.064$, Block 2 Adjusted $R^{2}=0.067$.

${ }^{\mathrm{a}}$ For the outcome of intentions to work with older adults, higher scores indicate more interest.

${ }^{\mathrm{b}}$ Yes/No questions coded as $1=$ yes, $2=$ no.

$* * p<0.01, * * * p<0.001$. 